\title{
Net force on an asymmetrically excited two-atom system from vacuum fluctuations
}

\author{
M. Donaire* \\ Laboratoire Kastler Brossel, UPMC-Sorbonnes Universités, CNRS, ENS-PSL Research University, Collège de France, 4, Place Jussieu, \\ F-75252 Paris, France \\ and Departamento de Física Teórica, Atómica y Óptica and IMUVA, Universidad de Valladolid, Paseo Belén 7, E-47011 Valladolid, Spain
}

(Received 26 April 2016; published 5 December 2016)

\begin{abstract}
A net force on a system of two dissimilar atoms, one of which is excited, is shown to result from their van der Waals interaction. It is accompanied by a net transfer of linear momentum to the quantum fluctuations of the electromagnetic field. This momentum results from the asymmetric interference of the virtual photons scattered off each atom along the interatomic direction, which is in itself a manifestation of the optical theorem. Ultimately, the virtual photons' momentum, of equal strength and opposite direction to the momentum gained by the two-atom system while excited, is released through directional spontaneous emission, which allows for an indirect measure, a posteriori, of the total force on the excited system. A quantitative prediction is made in a two-alkali atom system. It is conjectured that a net force and hence a nonzero momentum of quantum fluctuations take place in any asymmetrically excited system.
\end{abstract}

DOI: 10.1103/PhysRevA.94.062701

\section{INTRODUCTION}

In two recent publications [1,2] it has been found that, in a nondriven two-atom system with one atom excited, the van der Waals ( $v d W$ ) forces on each atom differ while actual photons are not yet emitted. This implies a nonvanishing net force upon the system. It is the purpose of this article to explain this phenomenon theoretically, to relate it to precedent findings, and to provide a quantitative estimate for its experimental verification. The explanation lies in the fundamental laws of momentum conservation and the optical theorem, from which we conjecture that a net force and hence a nonzero momentum of quantum fluctuations occur in any asymmetrically excited system.

VdW forces between neutral atoms are the result of the quantum fluctuations of both the electromagnetic (EM) field and the atomic charges $[3,4]$. At zero temperature, in the electric dipole approximation and for two atoms in their ground states located at a distance $R$ apart, the atoms undergo a series of virtual $E 1$ transitions to upper levels accompanied by the exchange of off-resonant virtual photons of frequency $\omega \lesssim c / R$. It is the mutual coupling of the charges of each atom to the virtual photons that induces correlations between their transient dipole moments, giving rise to a nonvanishing interaction that can be computed within the framework of stationary quantum perturbation theory. For short interatomic distances in comparison to the relevant transition wavelengths, the resultant forces are referred to as London dispersion forces [4-6]. For large distances they are known as CasimirPolder forces $[4,5,7]$. Off-resonant virtual photons mediate the transfer of linear momentum between the two atoms, being the momentum gained by each atom of equal strength and opposite sign.

The situation is different when at least one of the atoms is excited. In addition to the aforementioned interaction mediated by off-resonant photons, transitions to lower energy atomic

\footnotetext{
*donaire@1kb.upmc.fr; manuel.donaire@uva.es; mad37ster@ gmail.com
}

levels proceed through the coupling of the charges to resonant photons. This was first noticed by Wylie and Sipe [8] and calculated for a two-atom system in Refs. [9-11] using stationary perturbation theory. The question of whether this resonant interaction is monotonic or oscillatory in space has been the subject of a number of articles_cf. Refs. [1,2,9-15] and references therein. Recently, it has become clear that the resonant interaction is inherently time dependent, since it involves a nearly reversible and periodic excitation transfer between the two atoms. For nonidentical atoms, in the weak coupling regime, the probability of total excitation transfer is small, it is attenuated by the spontaneous emission of each atom in free space and its frequency is given by the detuning between the atomic species [1,12-14]. As shown in Refs. [1,2,12-15], the resonant interaction is to be computed within the framework of time-dependent perturbation theory, even though it is quasistationary for the usual case of an adiabatic excitation $[1,14]$.

Relevant to us is that only the strengths of the resonant forces on each atom differ [1,2]. It is a consequence of the fact that, in contrast to off-resonant photons, resonant virtual photons not only transfer momentum between the two atoms, but may also carry a net momentum off the two-atom system. This can be understood from the calculation of spontaneous emission rates, where resonant photons are the ones contributing to the probability of actual emission in Fermi's golden rule. In the first place, the probability of emission of a single atom in free space along any given direction equals at any time the probability of emission in the opposite direction. This is a consequence of the well-defined parity of the single-atom excited state, and implies that only when an actual photon is emitted a net momentum is carried by that photon, of uncertain direction a priori, which is accompanied by the corresponding recoil of the atom in the opposite direction. On the contrary, in the presence of an asymmetric environment, e.g., another nonexcited atom, the probability of emission is also asymmetric. This is a consequence of the non-well-defined parity of the two-atom excited state. We will show that it leads to a net and continuous transfer of momentum to the resonant virtual photons prior to 
actual emission. It is the nonvanishing rate of the recoil of the yet excited two-atom system that implies a nonvanishing net force upon the system.

The remainder of this paper is organized as follows. In Sec. II we describe the setup of the system and review the essentials of the approach in Ref. [1]. In Sec. III we prove the equivalence between the total force on the atoms and the time variation of the virtual photons' momentum. In Sec. IV we show that the nonvanishing of that momentum is a manifestation of the optical theorem. In Sec. V we propose directional spontaneous emission as an indirect measure, $a$ posteriori, of the net force on the atomic system. We finalize with the conclusions and comments of Sec. VI.

\section{NET FORCE ON THE TWO-ATOM SYSTEM}

Let us consider two atoms, $A$ and $B$, located a distance $R$ apart, with atom $A$ excited by a $\pi$ pulse. We are interested in the interatomic interaction at a certain time $T$ greater than the pulse duration and less than the lifetime of the excited state. Thus, the atoms are free to evolve and atom $A$ remains excited during the time of observation.

For simplicity, we take two two-level atoms of different types, with transition frequencies $\omega_{A}$ and $\omega_{B}$, and linewidths $\Gamma_{A}$ and $\Gamma_{B}$, respectively. Generally, the upper and lower states of each atom, $\left|A_{ \pm}\right\rangle,\left|B_{ \pm}\right\rangle$, may be degenerate. Further, in order to rest within the perturbative regime we ensure the distinction between the atomic species by demanding that the detuning $\Delta_{A B} \equiv \omega_{A}-\omega_{B}$ be such that $\left|\Delta_{A B}\right| \gg\langle W\rangle / \hbar,\left(\Gamma_{A}+\Gamma_{B}\right) / 2$, with $W$ being the interaction Hamiltonian. In Schrödinger's representation, the time propagator of the two-atom-EM field system reads, within a time interval $\left[T_{0}, T\right], \mathbb{U}\left(T-T_{0}\right)=$ $\mathcal{T} \exp \left[-i \hbar^{-1} \int_{T_{0}}^{T} d t\left(H_{0}+W\right)\right]$. In this expression $H_{0}$ is the sum of the free Hamiltonians of the internal atomic states and of the EM field, $H_{0}=\hbar \omega_{A}\left|A_{+}\right\rangle\left\langle A_{+}\left|+\hbar \omega_{B}\right| B_{+}\right\rangle\left\langle B_{+}\right|+$ $\sum_{\mathbf{k}, \epsilon} \hbar \omega\left(a_{\mathbf{k}, \epsilon}^{\dagger} a_{\mathbf{k}, \epsilon}+1 / 2\right)$, where $\omega=c k$ is the photon frequency, and the operators $a_{\mathbf{k}, \epsilon}^{\dagger}$ and $a_{\mathbf{k}, \epsilon}$ are the creation and annihilation operators of photons with momentum $\hbar \mathbf{k}$ and polarization $\epsilon$, respectively. Finally, the interaction Hamiltonian in the electric dipole approximation reads $W=W_{A}+W_{B}$, with $W_{A, B}=-\mathbf{d}_{A, B} \cdot \mathbf{E}\left(\mathbf{R}_{A, B}\right)$. In this expression $\mathbf{d}_{A, B}$ are the electric dipole operators of each atom and $\mathbf{E}\left(\mathbf{R}_{A, B}\right)$ is the electric field operator evaluated at the position of each atom, which can be written in the usual manner as a sum over normal modes,

$$
\begin{aligned}
\mathbf{E}\left(\mathbf{R}_{A, B}\right) & =\sum_{\mathbf{k}} \mathbf{E}_{\mathbf{k}}^{(-)}\left(\mathbf{R}_{A, B}\right)+\mathbf{E}_{\mathbf{k}}^{(+)}\left(\mathbf{R}_{A, B}\right) \\
& =i \sum_{\mathbf{k}, \epsilon} \sqrt{\frac{\hbar c k}{2 \mathcal{V} \epsilon_{0}}}\left[\epsilon a_{\mathbf{k}, \epsilon} e^{i \mathbf{k} \cdot \mathbf{R}_{A, B}}-\epsilon^{*} a_{\mathbf{k}, \epsilon}^{\dagger} e^{-i \mathbf{k} \cdot \mathbf{R}_{A, B}}\right],
\end{aligned}
$$

where $\mathcal{V}$ is a volume of quantization and $\mathbf{E}_{\mathbf{k}}^{(\mp)}$ denote the annihilation and creation electric field operators of photons of momentum $\hbar \mathbf{k}$, respectively. Correspondingly, we define $W_{A, B ; \mathbf{k}}^{(\mp)}=-\mathbf{d}_{A, B} \cdot \mathbf{E}_{\mathbf{k}}^{(\mp)}\left(\mathbf{R}_{A, B}\right)$.

Next, considering $W$ as a perturbation to the free Hamiltonians, the unperturbed time propagator for free atom and free photon states is, within the time interval $\left[t^{\prime}, t\right], \mathbb{U}_{0}\left(t-t^{\prime}\right)=$ $\exp \left[-i \hbar^{-1} H_{0}\left(t-t^{\prime}\right)\right]$. In terms of $W$ and $\mathbb{U}_{0}, \mathbb{U}\left(T-T_{0}\right)$ admits an expansion in powers of $W$ which can be developed out of the time-ordered exponential equation,

$\mathbb{U}\left(T-T_{0}\right)=\mathbb{U}_{0}(T) \mathcal{T} \exp \int_{T_{0}}^{T}(-i / \hbar) \mathbb{U}_{0}^{\dagger}(t) W \mathbb{U}_{0}\left(t-T_{0}\right) d t$

Denoting the term of order $W^{n}$ in the corresponding series by $\delta \mathbb{U}^{(n)}$, we may write $\mathbb{U}\left(T-T_{0}\right)=\mathbb{U}_{0}\left(T-T_{0}\right)+$ $\sum_{n=1}^{\infty} \delta \mathbb{U}^{(n)}\left(T-T_{0}\right)$. We denote by $\left|\Psi_{0}\right\rangle$ the state of the two-atom-EM field system at time $T_{0}$ after which the system is free to evolve with atom $A$ excited. Hereafter we will omit $T_{0}$ everywhere for simplicity, and we will omit $T$, unless necessary, in the argument of expectation values. Consequently, the state of the system at time $T \geqslant T_{0}$ reads $|\Psi(T)\rangle=\mathbb{U}(T)\left|\Psi_{0}\right\rangle$.

Straight application of the definition of the force operator on each atom yields for the total force on the atoms,

$$
\begin{aligned}
\left\langle\mathbf{F}_{A}+\mathbf{F}_{B}\right\rangle & =\left\langle\dot{\mathbf{Q}}_{A}+\dot{\mathbf{Q}}_{B}\right\rangle \\
& =-i \hbar \partial_{T}\left\langle\Psi_{0}\left|\mathbb{U}^{\dagger}(T)\left(\nabla_{\mathbf{R}_{A}}+\nabla_{\mathbf{R}_{B}}\right) \mathbb{U}(T)\right| \Psi_{0}\right\rangle \\
& =-\left\langle\nabla_{\mathbf{R}_{A}} W_{A}\right\rangle-\left\langle\nabla_{\mathbf{R}_{B}} W_{B}\right\rangle,
\end{aligned}
$$

where $\mathbf{Q}_{A, B}$ are the kinetic momentum operators of the centers of mass of each atom, and terms linear in $v_{A, B} / c$, with $v_{A, B}$ the velocities of the atoms, have been discarded [16].

The diagrammatical representation of $\left\langle W_{A, B}\right\rangle$ at $O\left(W_{A}^{2} W_{B}^{2}\right)$ was already given in Ref. [1]. In total, 24 terms, two per each of the 12 diagrams in Fig. 1, contribute to the force on each atom. It was argued in Sec. I that only those diagrams in which photons may resonate with the atomic transitions- cf. Ref. [13] — contribute to a nonzero total force on the system. In general, that force contains a number of terms which oscillate rapidly at the frequency of the detuning, $\Delta_{A B}$, together with a few quasistationary terms attenuated by a factor $e^{-\Gamma_{A} T}$ [1]. Further, it was shown explicitly in Ref. [1], and also anticipated in Ref. [14], that the fast oscillating terms vanish after an adiabatic excitation. In particular, only the two kinds of diagrams in Figs. $2\left(a_{1}\right), 2\left(a_{2}\right)$, and $2\left(b_{1}\right)-2\left(b_{3}\right)$ contribute to the resonant forces after an adiabatic excitation. Thus, they are the only ones responsible for the nonvanishing of Eq. (1).

For the usual case of an excitation driven by a $\pi$ pulse of frequency $\Omega$, with $\left|\Delta_{A B}\right| \gg \Omega \gg \Gamma_{A}$, the forces become quasistationary at times of observation $\Gamma_{A}^{-1} \gtrsim T \geqslant T_{0}=\pi / \Omega$. The addition of the forces on both atoms yields [1]

$$
\begin{aligned}
\left\langle\mathbf{F}_{A}+\mathbf{F}_{B}\right\rangle(T) \simeq & \left\langle\mathbf{F}_{A}+\mathbf{F}_{B}\right\rangle_{0} e^{-\Gamma_{A} T}=\mathcal{U}^{i j p q} e^{-\Gamma_{A} T} \\
& \times \nabla_{\mathbf{R}}\left[\operatorname{Im} G_{i j}^{(0)}\left(\mathbf{R}, \omega_{A}\right) \operatorname{Im} G_{p q}^{(0)}\left(\mathbf{R}, \omega_{A}\right)\right]
\end{aligned}
$$

In this equation $\mathbb{G}^{(0)}(\mathbf{R}, \omega)$ is the dyadic Green's function of the electric field induced at $\mathbf{R}=\left\langle\mathbf{R}_{A}-\mathbf{R}_{B}\right\rangle$ by an electric dipole of frequency $\omega=c k$,

$$
\mathbb{G}^{(0)}(\mathbf{R}, \omega)=\frac{k e^{i k R}}{-4 \pi}\left[\alpha / k R+i \beta /(k R)^{2}-\beta /(k R)^{3}\right],
$$

where the tensors $\alpha$ and $\beta$ read $\alpha=\mathbb{I}-\mathbf{R R} / R^{2}, \beta=\mathbb{I}-$ $3 \mathbf{R R} / R^{2}$, and $\mathcal{U}^{i j p q}=4 \omega_{B} k_{A}^{4} \mu_{A}^{i} \mu_{A}^{q} \mu_{B}^{j} \mu_{B}^{p} /\left[\epsilon_{0}^{2} \hbar\left(\omega_{A}^{2}-\omega_{B}^{2}\right)\right]$, with $\mu_{A}^{i}=\left\langle A_{-}\left|d_{A}^{i}\right| A_{+}\right\rangle, \mu_{B}^{j}=\left\langle B_{-}\left|d_{B}^{j}\right| B_{+}\right\rangle$. 


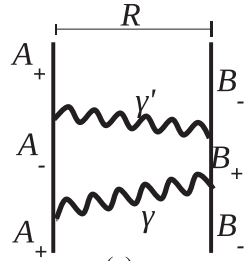

(a)

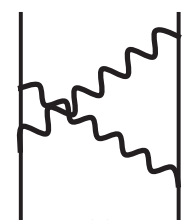

(g)

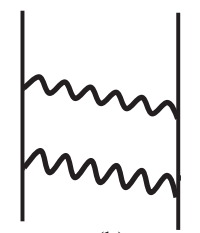

(b)

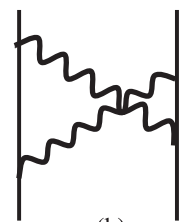

(h)

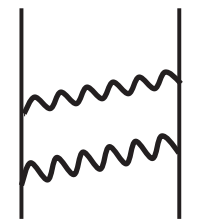

(c)

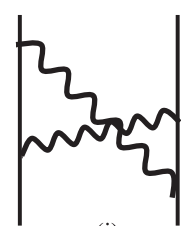

(i)

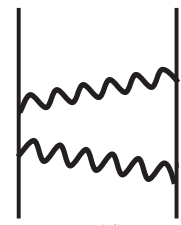

(d)

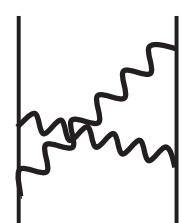

(j)

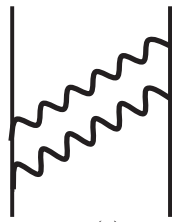

(e)

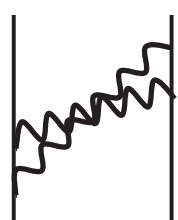

(k)

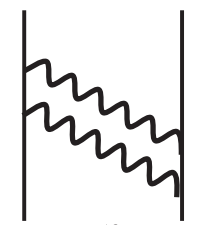

(f)

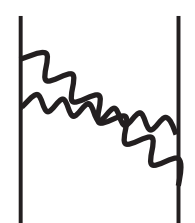

FIG. 1. Diagrammatic representation of the 12 time-ordered processes which contribute to the van der Waals interaction between atoms $A$ and $B$ at the lowest order in $W . R$ denotes the distance between the atoms. The time variable runs along the vertical.

\section{VIRTUAL PHOTONS' MOMENTUM}

In this section we prove that the momentum gained by the two-atom system while atom $A$ is excited is compensated by the transfer of an opposite momentum to the virtual photons exchanged by the atoms.

Translation invariance implies the conservation of total momentum in the two-atom-EM field system. Thus, a nonzero total force on the atoms must be compensated by an opposite variation of the momentum of the field. Since quantum field fluctuations couple to the atomic charges, the EM vacuum $\left|0_{\gamma}\right\rangle$ and the atomic states are entangled, $|\Psi\rangle=\left|A_{+}, B_{-}, 0_{\gamma}\right\rangle$, and net momentum may be transferred to the quantum vacuum fluctuations. In the following we show over the relevant diagrams that this is indeed the case. That is, being $\mathbf{P}=\sum_{\mathbf{k}, \epsilon} \hbar \mathbf{k}\left(a_{\mathbf{k}, \epsilon}^{\dagger} a_{\mathbf{k}, \epsilon}+1 / 2\right)$ the momentum operator of the EM field, we show that $\langle\dot{\mathbf{P}}\rangle=-\left\langle\mathbf{F}_{A}+\mathbf{F}_{B}\right\rangle$ at zero order in $v_{A, B} / c{ }^{1}$ Hence, a net force on the atomic system implies a nonzero momentum of the quantum fluctuations and vice versa.

Hereafter, we will refer to the quantities associated with the diagram of Figs. $2\left(\mathrm{a}_{1}\right)$ and $2\left(\mathrm{a}_{2}\right)$ as rotating wave ( $\left.r w\right)$, since they scale as $\sim \Delta_{A B}^{-1}$. Their contribution dominates under quasiresonant conditions, $\left|\Delta_{A, B}\right| \gg \omega_{A, B}$. Likewise, the quantities associated with the diagram of Figs. $2\left(b_{1}\right)-2\left(b_{3}\right)$ will be referred to as counter-rotating $(\mathrm{cr})$, as they go like $\sim\left(\omega_{A}+\omega_{B}\right)^{-1}$.

In the first place, the time derivative of the one-photon momentum associated with the rotating diagram reads

$$
\langle\dot{\mathbf{P}}\rangle_{r w}^{1 \gamma}=\sum_{\mathbf{k}} \partial_{T}\left\langle\Psi_{0}\left|\delta \mathbb{U}_{r w}^{\dagger(1)}(T, \mathbf{k}) \hbar \mathbf{k} \delta \mathbb{U}_{r w}^{(3)}(T, \mathbf{k})\right| \Psi_{0}\right\rangle+\text { c.c. },
$$

where the first term corresponds to the diagram on the left-hand side (lhs) of Fig. 2( $\left.a_{1}\right)$, and its complex conjugate (c.c.) does

\footnotetext{
${ }^{1}$ In addition to the transverse momentum $\mathbf{P}$ there exists also a longitudinal momentum operator [22] which, in the electric dipole approximation, equals the Röntgen momentum [16]. Consistently with the neglect of terms of order $v_{A, B} / c$ in the total force, it is discarded here.
}

to the diagram on the lhs of Fig. 2(a $\left.a_{2}\right)$. The expressions of the time propagators appearing in Eq. (4) and thereafter are compiled in Appendix A.

Performing the time derivative over the propagators we arrive at

$$
\begin{aligned}
\langle\dot{\mathbf{P}}\rangle_{r w}^{1 \gamma}= & \sum_{\mathbf{k}}\left[\left\langle\Psi_{0}\left|\mathbb{U}_{0}^{\dagger}(T) i \mathbf{k} W_{A, \mathbf{k}}^{(-)} \delta \mathbb{U}_{r w}^{(3)}(T, \mathbf{k})\right| \Psi_{0}\right\rangle\right. \\
& \left.+\left\langle\Psi_{0}\left|\delta \mathbb{U}_{r w}^{\dagger(1)}(T, \mathbf{k})(-i \mathbf{k}) W_{B, \mathbf{k}}^{(+)} \delta \mathbb{U}_{r w}^{(2)}(T)\right| \Psi_{0}\right\rangle\right]+ \text { c.c. } \\
= & \left\langle\nabla_{\mathbf{R}_{A}} W_{A}\right\rangle_{r w}+\left\langle\nabla_{\mathbf{R}_{B}} W_{B}\right\rangle_{r w} .
\end{aligned}
$$

The first two terms on the right-hand side (rhs) of Eq. (5) correspond to the two diagrams on the rhs of Fig. 2( $\left.a_{1}\right)$, respectively. Likewise, their complex conjugates correspond to the diagrams on the rhs of Fig. 2( $\left.a_{2}\right)$.

As for the time derivative of the EM momentum associated with the counter-rotating diagram, it contains both one-photon and two-photon components. The derivative of the one-photon momentum is

$$
\langle\dot{\mathbf{P}}\rangle_{c r}^{1 \gamma}=\sum_{\mathbf{k}} \partial_{T}\left\langle\Psi_{0}\left|\delta \mathbb{U}_{c r}^{\dagger(1)}(T, \mathbf{k}) \hbar \mathbf{k} \delta \mathbb{U}_{c r}^{(3)}(T, \mathbf{k})\right| \Psi_{0}\right\rangle+\text { c.c. }
$$

The first term on the rhs of Eq. (6) corresponds to the diagram on the lhs of Fig. 2(b $\left.b_{1}\right)$, while its complex conjugate corresponds to the diagram on the lhs of Fig. 2( $\left.b_{2}\right)$. Derivation with respect to $T$ yields

$$
\begin{aligned}
\langle\dot{\mathbf{P}}\rangle_{c r}^{1 \gamma}= & \sum_{\mathbf{k}}\left\langle\Psi_{0}\left|\mathbb{U}_{0}^{\dagger}(T) i \mathbf{k} W_{A, \mathbf{k}}^{(-)} \delta \mathbb{U}_{c r}^{(3)}(T, \mathbf{k})\right| \Psi_{0}\right\rangle \\
& +\mathcal{J}(T)+\text { c.c. }=\left\langle\nabla_{\mathbf{R}_{A}} W_{A}\right\rangle_{c r}+2 \operatorname{Re} \mathcal{J}(T) .
\end{aligned}
$$

The first term on the rhs of Eq. (7) corresponds to the diagram on the rhs of Fig. 2(b $\left.b_{1}\right)$, while its complex conjugate corresponds to the diagram on the rhs of Fig. 2( $\left.b_{2}\right)$. The function $\mathcal{J}(T)$ is given at the end of Appendix A, and is depicted by ellipsis in Fig. $2\left(b_{2}\right)$. Regarding the variation of the two-photon momentum,

$$
\begin{aligned}
\langle\dot{\mathbf{P}}\rangle_{c r}^{2 \gamma}= & \sum_{\mathbf{k}, \mathbf{k}^{\prime}} \partial_{T}\left\langle\Psi_{0}\right| \delta \mathbb{U}_{c r}^{\dagger(2)}\left(T, \mathbf{k}^{\prime}, \mathbf{k}\right) \hbar\left(\mathbf{k}+\mathbf{k}^{\prime}\right) \\
& \times \delta \mathbb{U}_{c r}^{(2)}\left(T, \mathbf{k}, \mathbf{k}^{\prime}\right)\left|\Psi_{0}\right\rangle
\end{aligned}
$$




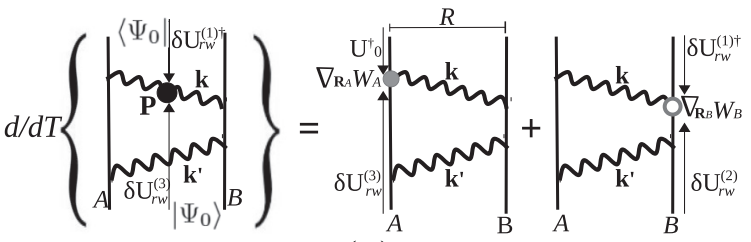

$\left(a_{1}\right)$
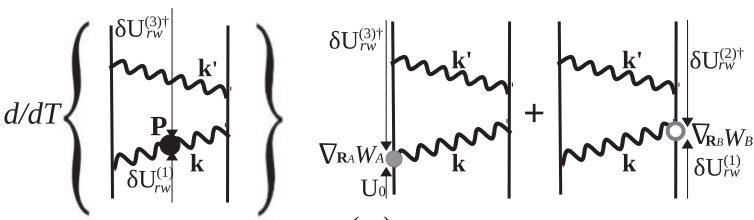

$\left(\mathbf{a}_{2}\right)$
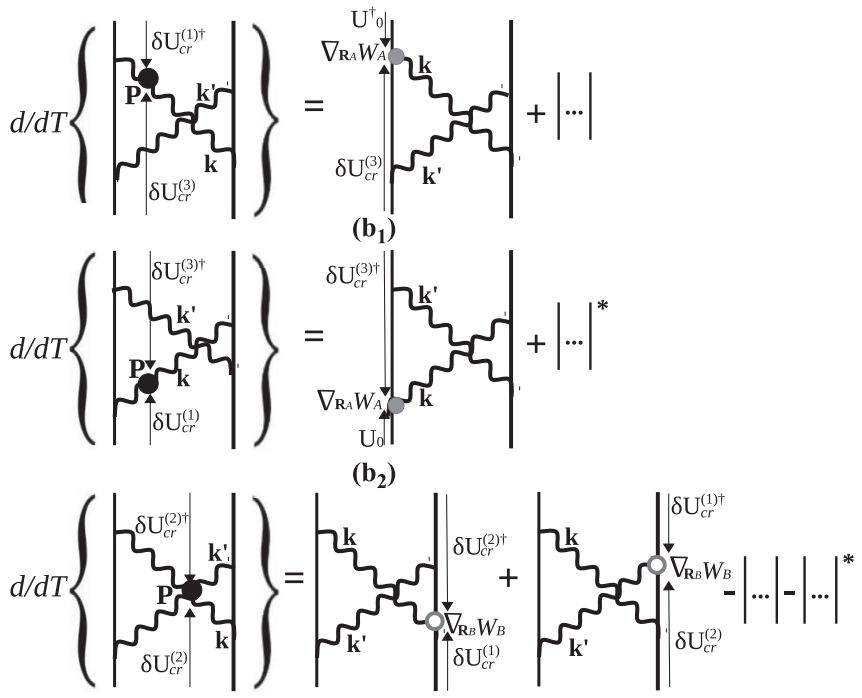

$\left(b_{3}\right)$

FIG. 2. Diagrammatic representation of Eqs. (5) [(a $\left.a_{1}\right)$ and $\left.\left(a_{2}\right)\right]$, (7) $\left[\left(b_{1}\right)\right.$ and $\left.\left(b_{2}\right)\right]$, and $(9)\left[\left(b_{3}\right)\right]$. Thick straight lines stand for time propagators of atomic states, while wavy lines are for space-time propagators of photons of momenta $\hbar \mathbf{k}$ and $\hbar \mathbf{k}^{\prime}$. The atoms are separated by a distance $R$ along the horizontal direction, whereas time runs along the vertical. Black circles stand for the insertion of an EM momentum operator $\mathbf{P}$, gray circles stand for $\nabla_{\mathbf{R}_{A}} W_{A}$, and white circles stand for $\nabla_{\mathbf{R}_{B}} W_{B}$. In each diagram two time propagators (depicted by vertical arrows) evolve the initial states, $\left|\psi_{0}\right\rangle$ and $\left\langle\psi_{0}\right|$, towards the observation time $T$, at which one of the aforementioned operators applies.

it is represented by the diagram on the lhs of Fig. 2(b $\left.b_{3}\right)$. Performing the time derivative of Eq. (8) we arrive at

$$
\begin{aligned}
\langle\dot{\mathbf{P}}\rangle_{c r}^{2 \gamma}= & \sum_{\mathbf{k}, \mathbf{k}^{\prime}}\left\langle\Psi_{0}\left|\delta \mathbb{U}_{c r}^{\dagger(2)}\left(T, \mathbf{k}^{\prime}, \mathbf{k}\right)(-i \mathbf{k}) W_{B, \mathbf{k}}^{(+)} \delta \mathbb{U}_{c r}^{(1)}(T, \mathbf{k})\right| \Psi_{0}\right\rangle \\
& -\mathcal{J}(T)+\text { c.c. }=\left\langle\nabla_{\mathbf{R}_{B}} W_{B}\right\rangle_{c r}-2 \operatorname{Re} \mathcal{J}(T) .
\end{aligned}
$$

The first term on the rhs of Eq. (9) is depicted by the first diagram on the rhs of Fig. 2( $\left.b_{3}\right)$, while its complex conjugate corresponds to the second diagram there. The term $-2 \operatorname{Re} \mathcal{J}(T)$ cancels the last term of Eq. (7), thus being of no relevance to us. Interestingly, the counter-rotating one-photon momentum compensates the gain of momentum by atom $A$, while the two-photon momentum compensates the momentum of atom
$B$. Equation (9), together with (7) and (5), complete the proof of the equality $\langle\dot{\mathbf{P}}\rangle=-\left\langle\mathbf{F}_{A}+\mathbf{F}_{B}\right\rangle$.

\section{THE OPTICAL THEOREM ON VIRTUAL PHOTONS}

Next, we show that a nonzero virtual photon's momentum, and hence a nonzero total force, are manifestations of the optical theorem. They result from the asymmetric interference of virtual photons along the interatomic direction.

It is apparent from the expressions on the rhs of Eqs. (4), (6), and (8) that the photonic momenta may be factored out of the time derivatives there. The remaining quantities are the partial emission rates, of one and two photons, which are not invariant under the transformation $\mathbf{k}^{\left({ }^{\prime}\right)} \rightarrow-\mathbf{k}^{\left({ }^{\prime}\right)}$. Hence, we can write

$$
\langle\dot{\mathbf{P}}\rangle=\sum_{\mathbf{k}, \mathbf{k}^{\prime}} \hbar \mathbf{k} \dot{\mathcal{P}}_{1 \gamma}(\mathbf{k})+\hbar\left(\mathbf{k}+\mathbf{k}^{\prime}\right) \dot{\mathcal{P}}_{2 \gamma}\left(\mathbf{k}, \mathbf{k}^{\prime}\right),
$$

with $\mathcal{P}_{1 \gamma}(\mathbf{k})=\left|\left\langle A_{-}, B_{-}, \gamma_{k}|\mathbb{U}(T)| \Psi_{0}\right\rangle\right|^{2}$ and $\mathcal{P}_{2 \gamma}\left(\mathbf{k}, \mathbf{k}^{\prime}\right)=$ $\left|\left\langle A_{-}, B_{-}, \gamma_{k}, \gamma_{k^{\prime}}|\mathbb{U}(T)| \Psi_{0}\right\rangle\right|^{2}$ being the probabilities of emission of one and two photons of momenta $\hbar \mathbf{k}$ and $\hbar\left(\mathbf{k}+\mathbf{k}^{\prime}\right)$, respectively. Mathematically, the noninvariance of the partial emission rates under $\mathbf{k}^{\left({ }^{\prime}\right)} \rightarrow-\mathbf{k}^{\left({ }^{\prime}\right)}$ is a consequence of the non-well-defined parity of the two-atom excited state. In the following and for simplicity we restrict ourselves to the quasiresonant approximation. Whereas the photons emitted from atom $A$ in free space do not distinguish between left and right along any direction [diagram of Fig. 3(a)], in the presence of atom $B$ there is a probability for them to be absorbed by $B$ and to be later scattered in any direction [Fig. 3(b)]. It may also happen that the photons be reabsorbed by $A$ and ultimately rescattered [Fig. 3(c)]. In addition, there are interference processes [Figs. 3(d)-3(g)]. The probabilities of scattering off $B$ and rescattering off $A$ are, however, of order $\Gamma_{A, B}^{2} / \Delta_{A B}^{2}$, and thus negligible in comparison to those of the interference terms which are $O\left(\Gamma_{A} / \Delta_{A B}\right)$. As shown by Berman in Ref. [17], the optical theorem demands that the total probability of the interference terms cancel out to guarantee probability conservation. ${ }^{2}$ That is, at leading order the interference of the photons emitted from $A$ in free space with the photons rescattered off $A$ in any direction [Figs. 3(d) and 3(e)] must be compensated by the interference of the photons emitted from $A$ with the photons scattered off $B$ along the interatomic direction $\mathbf{R}$ [Figs. 3(f) and 3(g)]. Since the location of the scatterer $B$ is obviously asymmetric with respect to the original emitter $A$, the interference along $\mathbf{R}$ is asymmetric, too, and so is the distribution of photonic momentum which enters Eq. (10). In particular, the contribution of diagrams Figs. 3(f) and 3(g) to $\mathcal{P}_{1 \gamma}(\mathbf{k})$ is, for asymptotic times and per unit solid angle-cf. Appendix B and Ref. [18],

$$
\operatorname{Re} \frac{k_{A}^{5} e^{i k_{A} R \cos \theta}}{\left(2 \pi \epsilon_{0} \hbar\right)^{2} \Gamma_{A} \Delta_{A B}} \mu_{A}^{i} \mu_{B}^{j}\left(\delta_{i j}-\hat{k}_{i} \hat{k}_{j}\right) \mu_{B}^{p} \mu_{A}^{q} G_{p q}^{(0)}\left(k_{A} R\right),
$$

where $\hat{\mathbf{k}}$ is a unitary vector along the emission direction and $\theta$ is the angle between $\hat{\mathbf{k}}$ and $\mathbf{R}$. In the far field, discarding

\footnotetext{
${ }^{2} \mathrm{~A}$ detailed proof of the optical theorem is given in Appendix B for asymptotic times in the quasiresonant approximation.
} 


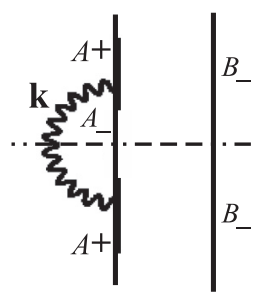

(a)

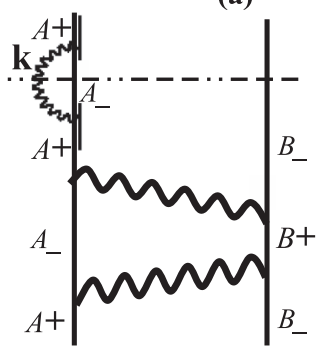

(d)

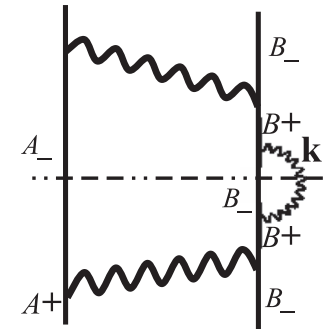

(b)

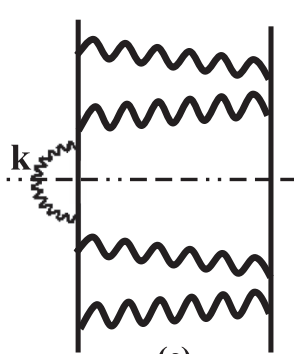

(c)

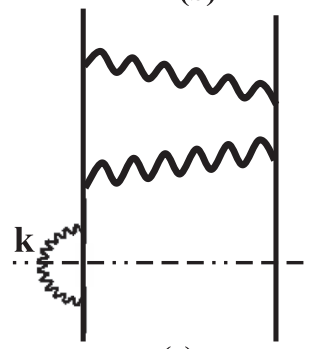

(e)

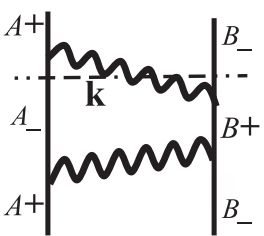

(f)

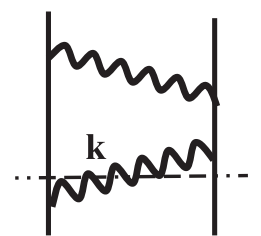

(g)

FIG. 3. Diagrammatic representation of $\mathcal{P}_{1 \gamma}(\mathbf{k})$ in the quasiresonant approximation. The horizontal dashed lines intersect the "virtually" emitted photon at the time of observation. Diagrams (d) and (e) result from the interference of (a) and (c), whereas (f) and (g) do so from (a) and (b).

the dependence on dipole orientations, this probability goes like $\sim \cos \left[k_{A} R(\cos \theta+1)\right] / k_{A} R$, which gives rise to a maximal differential probability between forward and backward emission at $R \approx 1.2 / k_{A}$.

\section{DIRECTIONAL SPONTANEOUS EMISSION}

Since radiation cannot be emitted continuously from the excited system, the momentum of the virtual photons increases until certain asymptotic time $T \rightarrow \infty \gtrsim \Gamma_{A}^{-1}$, at which $\langle\mathbf{P}\rangle_{\infty} \simeq-\left\langle\mathbf{F}_{A}+\mathbf{F}_{B}\right\rangle_{0} / \Gamma_{A}$, the total force of Eq. (2) vanishes, atom $A$ deexcites, and an actual photon is spontaneously emitted, carrying the momentum $\langle\mathbf{P}\rangle_{\infty}$. It is thus $\langle\mathbf{P}\rangle_{\infty}$ that generates the directionality of spontaneous emission along $\mathbf{R}$ [19]. It is worth noting that whereas the remaining momentum of the emitted photon is uncertain, its component $\langle\mathbf{P}\rangle_{\infty}$ is known beforehand, and so is the corresponding recoil of the atomic system. In other words, while for the case of nondirectional emission the recoil of the excited atom remains uncertain until the actual photon is emitted, the recoil of the atomic system due to directional spontaneous emission can be observed continuously evolving until actual emission takes place.

From the expression of $\langle\mathbf{P}\rangle_{\infty}$ in terms of the initial force $\left\langle\mathbf{F}_{A}+\mathbf{F}_{B}\right\rangle_{0}$, we conclude that an indirect measurement of that force can be obtained from the detection of directional emission a posteriori. An analogous expression for $\langle\mathbf{P}\rangle_{\infty}$ was obtained by Scheel et al. [20] for the force of an excited atom in close proximity to a nanofiber. Although nonreciprocity between the forces on the atom and on the nanofiber could occur in that system, its theoretical study is problematic since the calculation of the force on the nanofiber would need a Hamiltonian formulation of the microscopical interaction between the EM field and the fiber.

In the following, we compute the total force $\left\langle\mathbf{F}_{A}+\mathbf{F}_{B}\right\rangle_{0}$ of Eq. (2) on a system composed by an atom of ${ }^{87} \mathrm{Rb}$ in the state $5 P_{1 / 2}$ and a ${ }^{40} \mathrm{~K}$ atom in its ground state, together with the directionality of the subsequent spontaneous emission. The latter can be quantified through the excess of momentum of photons emitted along $\mathbf{R}, D=\mathbf{R} \cdot\langle\mathbf{P}\rangle_{\infty} c / R h$ [20]. In Fig. 4 we plot the values of $D$ together with the total force as a function of $R$. Maximum values are obtained for $R \simeq 1.28 / k_{A}$, where $D$ is $\sim 10$ times greater than $\Gamma_{A}$. The vdW potentials are also six orders of magnitude smaller than $D$ at the distance where the value of $D$ is maximum, thus the vdW shift on the frequency of the emitted photon is negligible there. Therefore, the accumulated phase shift of a photon emitted along the interatomic direction with respect to the accumulated phase of a photon emitted from atom $A$ in free space is solely due to $\langle\mathbf{P}\rangle_{\infty}$ in good approximation. That is, the phase shift accumulated between two points separated a distance $d$ and aligned with the interatomic radius would be $\delta \varphi= \pm 2 \pi d D / c$, where the sign in front depends on whether

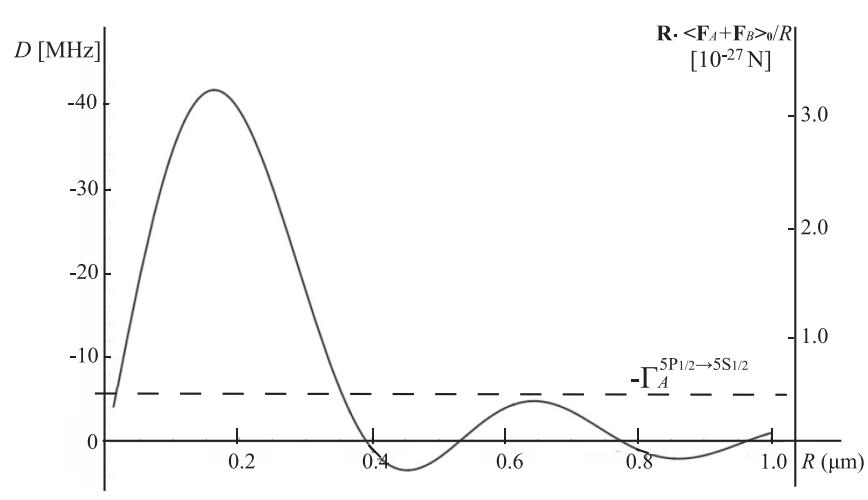

FIG. 4. Graphical representation, as a function of the interatomic distance $R$, of the directionality of spontaneous emission $D$, and of the total force on a system composed by an atom of ${ }^{87} \mathrm{Rb}$ in the state $5 P_{1 / 2}$ and a ground state ${ }^{40} \mathrm{~K}$ atom. 
the two points lie on the right- or on the left-hand side of the atomic system.

\section{CONCLUSIONS AND DISCUSSION}

In this article we have shown that the net vdW force acting on a nondriven two-atom system, with one atom excited, is compensated by the continuous transfer of momentum to quantum EM field fluctuations, in accordance with conservation of total momentum. The nonvanishing of the EM momentum, and hence of the net force, is a manifestation of the optical theorem on the scattering of virtual photons. Ultimately, when the system de-excites, the virtual photons' momentum is released through directional spontaneous emission and the classical action-reaction principle is restored, being zero the addition of the atomic momenta and the momentum of the actual photon emitted. Directional spontaneous emission is proposed as an indirect measure, a posteriori, of the total force on the excited system.

The spectroscopical measure of the phase shift $\delta \varphi$ is technically easier to perform than the direct measure of the forces on each atom. Nevertheless, the latter could be observed through the displacement experienced by each atom inside harmonic traps with respect to their equilibrium positions in the absence of the vdW interaction. The observation of nonreciprocity between the forces on each atom would be interesting, as it would prove the existence of a net recoil of the two-atom system prior to the emission of the actual photon.

A comment is in order concerning the concept of virtual photons' momentum. In the first place, in contrast to the EM momentum found in Refs. [21,22], where the asymmetry of the EM spectrum under $\mathbf{k} \rightarrow-\mathbf{k}$ is caused by the presence of magnetoelectric media and external fields, here it is a consequence of the optical theorem within an asymmetrically excited system. Nonetheless, it is the noninvariance under parity and time reversal that allows for a virtual photons' momentum in both kinds of systems. On the other hand, Feigel [21] coined the term vacuum momentum to refer to the momentum of virtual photons, since those photons are the quantum fluctuations of the EM field in a state with no actual photons but entangled with atomic states, as it is the case of our state $|\Psi\rangle$. In the context of the so-called Casimir physics, that EM state is commonly referred to as dressed vacuum, and the corresponding field fluctuations are called vacuum fluctuations. The nature of those fluctuations is identical to that of the vacuum fluctuations which are invoked to explain the Lamb shift and spontaneous emission in free space. Therefore, in that context, the virtual photons' momentum calculated in the present article can be equally referred to as vacuum momentum.

Despite the fact that our calculation restricts to the vdW forces between two dissimilar atoms in the perturbative regime, the phenomena described in this article also hold in a system of two identical atoms with one of them excited, for the latter is a noninvariant system under parity, too. Hence, the directionality of spontaneous emission from such a system was found by Berman in Ref. [18].

Finally, beside our simple QED calculation, momentum conservation and the optical theorem manifest themselves in any quantum field theory as a result of translation invariance, locality, and unitarity. Therefore, a nonzero vacuum momentum and hence a nonvanishing total force are to be found in any asymmetrically excited system. For instance, that is the case of an heterogeneous system of unstable fundamental particles, charged with respect to a common symmetry group, whose decays proceed through the emission of particles associated with the symmetry. It is left for a separate publication the calculation of a net nuclear force in a two-nucleus system, with one of the nucleus unstable against beta decay.

\section{ACKNOWLEDGMENTS}

We thank P. Berman M.-P. Gorza, S. Y. Buhmann, P. Barcellona, S. Scheel, B. Gouraud, and M. Mukherjee for useful discussions on this problem. Financial support from French Contracts No. ANR-10-IDEX-0001-02-PSL and No. ANR-13-BS04-0003-02, and from Spanish Grants No. MTM2014-57129-C2-1-P (MINECO) and No. VA057U16 (Junta de Castilla y León) is gratefully acknowledged.

\section{APPENDIX A: TIME PROPAGATORS IN ROTATING AND COUNTER-ROTATING PROCESSES}

We give below the explicit equations for the time-propagator components of order $n, \delta \mathbb{U}_{r w}^{(n)}$, and $\delta \mathbb{U}_{c r}^{(n)}$, which enter Eqs. (4)-(9) in Sec. II for the time derivative of the vacuum momentum, and appear on the rotating and counter-rotating diagrams of Fig. 2. As for Eq. (4) and Fig. $2\left(\mathrm{a}_{1}\right), \delta \mathbb{U}_{r w}^{\dagger(1)}(T, \mathbf{k})$ and $\delta \mathbb{U}_{r w}^{(3)}(T, \mathbf{k})$ read, respectively,

$$
\begin{aligned}
\delta \mathbb{U}_{r w}^{\dagger(1)}(T, \mathbf{k}) & =(i / \hbar) \int_{0}^{T} d t \mathbb{U}_{0}^{\dagger}(t) W_{A, \mathbf{k}}^{(-)} \mathbb{U}_{0}^{\dagger}(T-t), \\
\delta \mathbb{U}_{r w}^{(3)}(T, \mathbf{k}) & =(-i / \hbar)^{3} \sum_{\mathbf{k}^{\prime}} \int_{0}^{T} d t \int_{0}^{t} d t^{\prime} \int_{0}^{t^{\prime}} d t^{\prime \prime} \mathbb{U}_{0}(T-t) W_{B, \mathbf{k}}^{(+)} \mathbb{U}_{0}\left(t-t^{\prime}\right) W_{B, \mathbf{k}^{\prime}}^{(-)} \mathbb{U}_{0}\left(t^{\prime}-t^{\prime \prime}\right) W_{A, \mathbf{k}^{\prime}}^{(+)} \mathbb{U}_{0}\left(t^{\prime \prime}\right) .
\end{aligned}
$$

The time derivative of the above quantities within Eq. (4) gives rise to Eq. (5), where the time propagator $\delta \mathbb{U}_{r w}^{(2)}(T)$ is

$$
\delta \mathbb{U}_{r w}^{(2)}(T)=(-i / \hbar)^{2} \sum_{\mathbf{k}^{\prime}} \int_{0}^{T} d t \int_{0}^{t} d t^{\prime} \mathbb{U}_{0}(T-t) W_{B, \mathbf{k}^{\prime}}^{(-)} \mathbb{U}_{0}\left(t-t^{\prime}\right) W_{A, \mathbf{k}^{\prime}}^{(+)} \mathbb{U}_{0}\left(t^{\prime}\right) .
$$


As for Eq. (6) and Fig. $2\left(\mathrm{~b}_{1}\right), \delta \mathbb{U}_{c r}^{\dagger(1)}(T, \mathbf{k})=\delta \mathbb{U}_{r w}^{\dagger(1)}(T, \mathbf{k})$, and $\delta \mathbb{U}_{c r}^{(3)}(T, \mathbf{k})$ is

$$
\delta \mathbb{U}_{c r}^{(3)}(T, \mathbf{k})=(-i / \hbar)^{3} \sum_{\mathbf{k}^{\prime}} \int_{0}^{T} d t \int_{0}^{t} d t^{\prime} \int_{0}^{t^{\prime}} d t^{\prime \prime} \mathbb{U}_{0}(T-t) W_{B, \mathbf{k}^{\prime}}^{(-)} \mathbb{U}_{0}\left(t-t^{\prime}\right) W_{B, \mathbf{k}}^{(+)} \mathbb{U}_{0}\left(t^{\prime}-t^{\prime \prime}\right) W_{A, \mathbf{k}^{\prime}}^{(+)} \mathbb{U}_{0}\left(t^{\prime \prime}\right) .
$$

Finally, the time propagator $\delta \mathbb{U}_{c r}^{(2)}\left(T, \mathbf{k}, \mathbf{k}^{\prime}\right)$ entering Eq. (8) and Fig. 2( $\left.\mathrm{b}_{3}\right)$ reads

$$
\delta \mathbb{U}_{c r}^{(2)}\left(T, \mathbf{k}, \mathbf{k}^{\prime}\right)=(-i / \hbar)^{2} \int_{0}^{T} d t \int_{0}^{t} d t^{\prime} \mathbb{U}_{0}(T-t) W_{B, \mathbf{k}}^{(+)} \mathbb{U}_{0}\left(t-t^{\prime}\right) W_{A, \mathbf{k}^{\prime}}^{(+)} \mathbb{U}_{0}\left(t^{\prime}\right) .
$$

Note that the propagator $\delta \mathbb{U}_{c r}^{\dagger(2)}\left(T, \mathbf{k}^{\prime}, \mathbf{k}\right)$ in Eqs. (8) and (9) is the Hermitian conjugate of the above expression upon which the exchange $\mathbf{k} \leftrightarrow \mathbf{k}^{\prime}$ has been performed.

As for the function $\mathcal{J}(T)$, which enters Eqs. (7) and (9) with opposite signs, it results from the time derivatives of $\delta \mathbb{U}_{c r}^{(3)}(T, \mathbf{k})$ within Eq. (6) and of $\delta \mathbb{U}_{c r}^{\dagger(2)}\left(T, \mathbf{k}^{\prime}, \mathbf{k}\right)$ within Eq. (8), respectively. It reads

$$
\mathcal{J}(T)=-\sum_{\mathbf{k}, \mathbf{k}^{\prime}}\left\langle\Psi_{0}\left|\delta \mathbb{U}_{r w}^{\dagger(1)}(T, \mathbf{k}) i \mathbf{k} W_{B, \mathbf{k}^{\prime}}^{(-)} \delta \mathbb{U}_{c r}^{(2)}\left(T, \mathbf{k}, \mathbf{k}^{\prime}\right)\right| \Psi_{0}\right\rangle
$$

\section{APPENDIX B: OPTICAL THEOREM IN THE QUASIRESONANT APPROXIMATION}

It has been argued in Sec. IV that satisfaction of the optical theorem guaranties the conservation of total emission probability. In the quasiresonant approximation, the leading order processes which contribute to the one-photon emission probability $\mathcal{P}_{1 \gamma}$ are represented diagrammatically in Fig. 3. We show next that, at leading order in $\Gamma_{A, B} / \Delta_{A B}, \mathcal{P}_{1 \gamma}=1$. This result was first obtained by Berman in Ref. [17] using an equivalent formalism.

In the first place, the probability of emission in free space reads, from diagram Fig. 3(a),

$$
\begin{aligned}
\mathcal{P}_{1 \gamma}^{(a)} & =\sum_{\mathbf{k}} \mathcal{P}_{1 \gamma}^{(a)}(\mathbf{k})=\sum_{\mathbf{k}}\left\langle\Psi_{0}\left|\delta \mathbb{U}_{r w}^{\dagger(1)}(T, \mathbf{k}) \delta \mathbb{U}_{r w}^{(1)}(T, \mathbf{k})\right| \Psi_{0}\right\rangle \\
& =\mu_{A}^{i} \mu_{A}^{j} \hbar^{-2} \sum_{\mathbf{k}}\left|\int_{0}^{T} d t e^{-i \omega(T-t)} e^{-i t \omega_{A}} e^{-t \Gamma_{A} / 2}\right|^{2}\left\langle 0_{\gamma}\left|E_{\mathbf{k}, i}^{(-)}\left(\mathbf{R}_{A}\right) E_{\mathbf{k}, j}^{(+)}\left(\mathbf{R}_{A}\right)\right| 0_{\gamma}\right\rangle .
\end{aligned}
$$

Performing the time integration, passing the sum over $\mathbf{k}$ to a continuous integral and performing the resultant integration over $\mathbf{k}$, we obtain for asymptotic times $\Gamma_{A} T \gg 1$,

$$
\mathcal{P}_{1 \gamma}^{(a)} \simeq \frac{-2 k_{A}^{2}}{\epsilon_{0} \hbar \Gamma_{A}} \mu_{A}^{i} \mu_{A}^{j} \operatorname{Im} G_{i j}^{(0)}\left(\mathbf{r}, \omega_{A}\right)=1, \quad \text { for } \mathbf{r} \rightarrow \mathbf{0},
$$

where in the last equality we have identified $\Gamma_{A}$, by consistency, with the free-space emission rate. Therefore, probability conservation demands the cancellation at the lowest order of the total emission coming from the rest of the diagrams in Fig. 3.

As for the emission probability of diagrams Figs. 2(d) and 2(e), which result from the interference of Figs. 3(a) and 3(c), we find for asymptotic times,

$$
\begin{aligned}
\mathcal{P}_{1 \gamma}^{(d, e)}= & \sum_{\mathbf{k}} \mathcal{P}_{1 \gamma}^{(c, d)}(\mathbf{k})=2 \operatorname{Re} \mu_{A}^{i} \mu_{A}^{j} \mu_{A}^{p} \mu_{B}^{q} \mu_{B}^{r} \mu_{A}^{s} \hbar^{-6} \sum_{\mathbf{k}, \mathbf{k}^{\prime}, \mathbf{k}^{\prime \prime}} \int_{0}^{T} d t \int_{0}^{T} d t^{\prime} \int_{0}^{t^{\prime}} d t^{\prime \prime} \int_{0}^{t^{\prime \prime}} d t^{\prime \prime \prime} \int_{0}^{t^{\prime \prime \prime}} d t^{i v} \int_{0}^{t^{i v}} d t^{v} e^{i \omega_{A} t} e^{-t \Gamma_{A} / 2} \\
& \times e^{-i\left(t-t^{\prime}\right) \omega} e^{-i\left(t^{\prime}-t^{\prime \prime}\right) \omega_{A}} e^{-\left(t^{\prime}-t^{\prime \prime}\right) \Gamma_{A} / 2} e^{-i\left(t^{\prime \prime}-t^{\prime \prime \prime}\right) \omega^{\prime}} e^{-i\left(t^{\prime \prime \prime}-t^{i v}\right) \omega_{B}} e^{-\left(t^{\prime \prime \prime}-t^{i v}\right) \Gamma_{B} / 2} e^{-i\left(t^{i v}-t^{v}\right) \omega^{\prime \prime}} e^{-i t^{v} \omega_{A}} e^{-t^{v} \Gamma_{A} / 2} \\
& \times\left\langle 0_{\gamma}\left|E_{\mathbf{k}, i}^{(-)}\left(\mathbf{R}_{A}\right) E_{\mathbf{k}, j}^{(+)}\left(\mathbf{R}_{A}\right)\right| 0_{\gamma}\right\rangle\left\langle 0_{\gamma}\left|E_{\mathbf{k}^{\prime}, p}^{(-)}\left(\mathbf{R}_{A}\right) E_{\mathbf{k}^{\prime}, q}^{(+)}\left(\mathbf{R}_{B}\right)\right| 0_{\gamma}\right\rangle\left\langle 0_{\gamma}\left|E_{\mathbf{k}^{\prime \prime}, r}^{(-)}\left(\mathbf{R}_{B}\right) E_{\mathbf{k}^{\prime \prime}, s}^{(+)}\left(\mathbf{R}_{A}\right)\right| 0_{\gamma}\right\rangle \\
& \simeq \frac{4 k_{A}^{4}}{\epsilon_{0}^{2} \hbar^{2} \Gamma_{A} \Delta_{A B}} \mu_{A}^{p} \operatorname{Im} G_{p q}^{(0)}\left(\mathbf{R}, \omega_{A}\right) \mu_{B}^{q} \mu_{B}^{r} \operatorname{Re} G_{r s}^{(0)}\left(\mathbf{R}, \omega_{A}\right) \mu_{A}^{s} .
\end{aligned}
$$

On the other hand, the partial emission probability of one photon of momentum $\hbar k_{A}$ along a generic direction $\hat{\mathbf{k}}$, given by Eq. (11), derives from the partial integration of the emission probability of diagrams Figs. 3(f) and 3(g), which are the result of the interference of the photons emitted in diagrams Figs. 3(a) and 3(b) along R. The expression for the total emission probability of diagrams Figs. 3(f) and $3(\mathrm{~g})$ is

$$
\begin{aligned}
\mathcal{P}_{1 \gamma}^{(f, g)} & =\sum_{\mathbf{k}} \mathcal{P}_{1 \gamma}^{(f, g)}(\mathbf{k})=2 \operatorname{Re} \sum_{\mathbf{k}}\left\langle\Psi_{0}\left|\delta \mathbb{U}_{r w}^{\dagger(1)}(T, \mathbf{k}) \delta \mathbb{U}_{r w}^{(3)}(T, \mathbf{k})\right| \Psi_{0}\right\rangle \\
& =-2 \operatorname{Re} \mu_{A}^{p} \mu_{B}^{q} \mu_{B}^{r} \mu_{A}^{s} \hbar^{-4} \sum_{\mathbf{k}, \mathbf{k}^{\prime}} \int_{0}^{T} d t \int_{0}^{T} d t^{\prime} \int_{0}^{t^{\prime}} d t^{\prime \prime} \int_{0}^{t^{\prime \prime}} d t^{\prime \prime \prime} e^{i \omega_{A} t} e^{-t \Gamma_{A} / 2} e^{-i\left(t-t^{\prime}\right) \omega} e^{-i\left(t^{\prime}-t^{\prime \prime}\right) \omega_{B}} e^{-\left(t^{\prime}-t^{\prime \prime}\right) \Gamma_{B} / 2}
\end{aligned}
$$




$$
\begin{aligned}
& \times e^{-i\left(t^{\prime \prime}-t^{\prime \prime \prime}\right) \omega^{\prime}} e^{-i t^{\prime \prime \prime} \omega_{A}} e^{-t^{\prime \prime} \Gamma_{A} / 2}\left\langle 0_{\gamma}\left|E_{\mathbf{k}, p}^{(-)}\left(\mathbf{R}_{A}\right) E_{\mathbf{k}, q}^{(+)}\left(\mathbf{R}_{B}\right)\right| 0_{\gamma}\right\rangle\left\langle 0_{\gamma}\left|E_{\mathbf{k}^{\prime}, r}^{(-)}\left(\mathbf{R}_{B}\right) E_{\mathbf{k}^{\prime}, s}^{(+)}\left(\mathbf{R}_{A}\right)\right| 0_{\gamma}\right\rangle \\
\simeq & \frac{-4 k_{A}^{4}}{\epsilon_{0}^{2} \hbar^{2} \Gamma_{A} \Delta_{A B}} \mu_{A}^{p} \operatorname{Im} G_{p q}^{(0)}\left(\mathbf{R}, \omega_{A}\right) \mu_{B}^{q} \mu_{B}^{r} \operatorname{Re} G_{r s}^{(0)}\left(\mathbf{R}, \omega_{A}\right) \mu_{A}^{s},
\end{aligned}
$$

from which the relation $\mathcal{P}_{1 \gamma}^{(d, e)}+\mathcal{P}_{1 \gamma}^{(f, g)}=0$ holds as anticipated.

Finally, we show for the sake of completeness that the total probabilities of scattering off atom $B$ and rescattering off atom $A$ in any direction, depicted by the diagrams Figs. 3(b) and 3(c), respectively, are of an order $\Gamma_{A, B} / \Delta_{A B}$ smaller than the previous ones. As for scattering off $B$, its probability is

$$
\begin{aligned}
\mathcal{P}_{1 \gamma}^{(b)}= & \sum_{\mathbf{k}} \mathcal{P}_{1 \gamma}^{(b)}(\mathbf{k})=\sum_{\mathbf{k}}\left\langle\Psi_{0}\left|\delta \mathbb{U}_{r w}^{\dagger(3)}(T, \mathbf{k}) \delta \mathbb{U}_{r w}^{(3)}(T, \mathbf{k})\right| \Psi_{0}\right\rangle \\
= & \mu_{B}^{i} \mu_{B}^{j} \hbar^{-6} \sum_{\mathbf{k}} \mid \sum_{\mathbf{k}^{\prime}} \int_{0}^{T} d t \int_{0}^{t} d t^{\prime} \int_{0}^{t^{\prime}} d t^{\prime \prime} e^{-i(T-t) \omega} e^{-i\left(t-t^{\prime}\right) \omega_{B}} e^{-\left(t-t^{\prime}\right) \Gamma_{B} / 2} e^{-i\left(t^{\prime}-t^{\prime \prime}\right) \omega^{\prime}} e^{-i t^{\prime \prime} \omega_{A}} e^{-t^{\prime \prime} \Gamma_{A} / 2} \\
& \times\left.\mu_{B}^{p} \mu_{A}^{q}\left\langle 0_{\gamma}\left|E_{\mathbf{k}^{\prime}, p}^{(-)}\left(\mathbf{R}_{B}\right) E_{\mathbf{k}^{\prime}, q}^{(+)}\left(\mathbf{R}_{A}\right)\right| 0_{\gamma}\right\rangle\right|^{2}\left\langle 0_{\gamma}\left|E_{\mathbf{k}, i}^{(-)}\left(\mathbf{R}_{B}\right) E_{\mathbf{k}, j}^{(+)}\left(\mathbf{R}_{B}\right)\right| 0_{\gamma}\right\rangle \\
\simeq & \frac{\mu_{A}^{p} \mu_{B}^{q} \mu_{B}^{r} \mu_{A}^{s}}{\epsilon_{0}^{2} \hbar^{2} \Delta_{A B}^{2}}\left[\frac{\left|\mu_{B}\right|^{2}}{\left|\mu_{A}\right|^{2}} k_{A}^{4} G_{p q}^{(0)}\left(\mathbf{R}, \omega_{A}\right) G_{r s}^{*(0)}\left(\mathbf{R}, \omega_{A}\right)+k_{B}^{4} G_{p q}^{(0)}\left(\mathbf{R}, \omega_{B}\right) G_{r s}^{*(0)}\left(\mathbf{R}, \omega_{B}\right)\right],
\end{aligned}
$$

and for rescattering off $A$ we find

$$
\begin{aligned}
\mathcal{P}_{1 \gamma}^{(c)}= & \sum_{\mathbf{k}} \mathcal{P}_{1 \gamma}^{(c)}(\mathbf{k})=\mu_{A}^{i} \mu_{A}^{j} \hbar^{-10} \sum_{\mathbf{k}} \mid \sum_{\mathbf{k}^{\prime}, \mathbf{k}^{\prime \prime}} \int_{0}^{T} d t^{\prime} \int_{0}^{t^{\prime}} d t^{\prime \prime} \int_{0}^{t^{\prime \prime}} d t^{\prime \prime \prime} \int_{0}^{t^{\prime \prime \prime}} d t^{i v} \int_{0}^{t^{i v}} d t^{v} \\
& \times e^{-i\left(T-t^{\prime}\right) \omega} e^{-i\left(t^{\prime}-t^{\prime \prime}\right) \omega_{A}} e^{-\left(t^{\prime}-t^{\prime \prime}\right) \Gamma_{A} / 2} e^{-i\left(t^{\prime \prime}-t^{\prime \prime \prime}\right) \omega^{\prime}} e^{-i\left(t^{\prime \prime \prime}-t^{i v}\right) \omega_{B}} e^{-\left(t^{\prime \prime \prime}-t^{i v}\right) \Gamma_{B} / 2} e^{-i\left(t^{i v}-t^{v}\right) \omega^{\prime \prime}} e^{-i t^{v} \omega_{A}} e^{-t^{v} \Gamma_{A} / 2} \\
& \times\left.\mu_{A}^{p} \mu_{B}^{q} \mu_{B}^{r} \mu_{A}^{s}\left\langle 0_{\gamma}\left|E_{\mathbf{k}^{\prime}, p}^{(-)}\left(\mathbf{R}_{A}\right) E_{\mathbf{k}^{\prime}, q}^{(+)}\left(\mathbf{R}_{B}\right)\right| 0_{\gamma}\right\rangle\left\langle 0_{\gamma}\left|E_{\mathbf{k}^{\prime \prime}, r}^{(-)}\left(\mathbf{R}_{B}\right) E_{\mathbf{k}^{\prime \prime}, s}^{(+)}\left(\mathbf{R}_{A}\right)\right| 0_{\gamma}\right\rangle\right|^{2}\left\langle 0_{\gamma}\left|E_{\mathbf{k}, i}^{(-)}\left(\mathbf{R}_{A}\right) E_{\mathbf{k}, j}^{(+)}\left(\mathbf{R}_{A}\right)\right| 0_{\gamma}\right\rangle \\
\simeq & 2\left[\frac{\mu_{A}^{p} \mu_{B}^{q} \mu_{B}^{r} \mu_{A}^{s}}{\epsilon_{0}^{2} \hbar^{2} \Gamma_{A} \Delta_{A B}} k_{A}^{4} G_{p q}^{(0)}\left(\mathbf{R}, \omega_{A}\right) G_{r s}^{*(0)}\left(\mathbf{R}, \omega_{A}\right)\right]^{2} .
\end{aligned}
$$

Both probabilities are $\mathcal{O}\left(\Gamma_{A, B}^{2} / \Delta_{A B}^{2}\right)$ as anticipated. They must be compensated by interference processes of an order higher than $\mathcal{P}_{1 \gamma}^{(d, e)}$ and $\mathcal{P}_{1 \gamma}^{(f, g)}$ in virtue of the optical theorem. Note, however, that for the case of identical atoms the emission probability $\mathcal{P}_{1 \gamma}^{(b)}$ is of the same order as that of $\mathcal{P}_{1 \gamma}^{(d, e)}$ and $\mathcal{P}_{1 \gamma}^{(f, g)}$. Hence, it is in that case the $\operatorname{sum} \mathcal{P}_{1 \gamma}^{(b)}+\mathcal{P}_{1 \gamma}^{(d, e)}+\mathcal{P}_{1 \gamma}^{(f, g)}$ that vanishes [18]—the term $\mathcal{P}_{1 \gamma}^{(d, e)}$ and part of $\mathcal{P}_{1 \gamma}^{(f, g)}$ are, however, missing in Ref. [18].

[1] M. Donaire, Phys. Rev. A 93, 052706 (2016).

[2] P. Barcellona, R. Passante, L. Rizzuto, and S. Y. Buhmann, Phys. Rev. A 94, 012705 (2016).

[3] C. Cohen-Tannoudji, J. Dupont-Roc, and G. Grynberg, Photons and Atoms. Introduction to Quantum Electrodynamics (WileyVCH Verlag, Weinheim, 2004).

[4] P. W. Milonni, The Quantum Vacuum (Academic Press, San Diego, 1994).

[5] D. P. Craig and T. Thirunamachandran, Molecular Quantum Electrodynamics (Dover, New York, 1998).

[6] F. London, Z. Phys. 63, 245 (1930).

[7] H. B. G. Casimir and D. Polder, Phys. Rev. 73, 360 (1948).

[8] J. M. Wylie and J. E. Sipe, Phys. Rev. A 30, 1185 (1984); 32, 2030 (1985).

[9] E. A. Power and T. Thirunamachandran, Phys. Rev. A 51, 3660 (1995).

[10] R. R. McLone and E. A. Power, Proc. R. Soc. London A 286, 573 (1965).

[11] L. Gomberoff, R. R. McLone, and E. A. Power, J. Chem. Phys. 44, 4148 (1966).
[12] P. W. Milonni and Seyed Mohammad Hashemi Rafsanjani, Phys. Rev. A 92, 062711 (2015).

[13] M. Donaire, R. Guérout, and A. Lambrecht, Phys. Rev. Lett. 115, 033201 (2015).

[14] P. R. Berman, Phys. Rev. A 91, 042127 (2015).

[15] Y. Sherkunov, Phys. Rev. A 75, 012705 (2007).

[16] M. Donaire and A. Lambrecht, Phys. Rev. A 93, 022701 (2016).

[17] P. R. Berman, Phys. Rev. A 76, 043816 (2007).

[18] P. R. Berman, Phys. Rev. A 55, 4466 (1997).

[19] R. H. Lehmberg, Phys. Rev. A 2, 889 (1970); Z. Ficek, R. Tanaś, and S. Kielich, Physica A 146, 452 (1987); V. E. Lembessis, A. A. Rsheed, O. M. Aldossary, and Z. Ficek, Phys. Rev. A 88, 053814 (2013).

[20] S. Scheel, S. Y. Buhmann, C. Clausen, and P. Schneeweiss, Phys. Rev. A 92, 043819 (2015).

[21] A. Feigel, Phys. Rev. Lett. 92, 020404 (2004).

[22] S. Kawka and B. A. van Tiggelen, Europhys. Lett. 89, 11002 (2010); M. Donaire, B. A. van Tiggelen, and G. L. J. A. Rikken, Phys. Rev. Lett. 111, 143602 (2013); J. Phys. Condens. Matter 27, 214002 (2015). 